\title{
Fetal Programming in Maternal Obesity
}

\author{
Philma Glora Muthuraj, Sathish Kumar Natarajan*
}

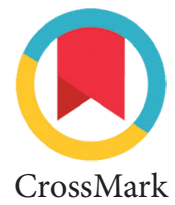

\section{ABSTRACT}

The nutritional status of the pregnant mother has a significant effect on the developing fetus which could be reflected as different forms of metabolic disorders in their postnatal life know as fetal programming. Recently obesity has became an epidemic and obese pregnant mothers have been shown to develop an adverse in utero environment as a consequence of chronic low-grade inflammation. This can have an significant impact on several organs including the placenta which connects the mother and the developing vital organs of the fetus such as heart, liver, immune system, and digestive system. Critical developmental processes can be affected in fetal organs that could compromise their efficient function during post-natal life. Overall, obese mothers are not only at risk of developing metabolic disorders on their own but also lead to deteriorating effects in the form of metabolic disorders even in their progeny at later parts of their lives. This again reiterates the fact that the health status of the parents is equally important for the wellbeing of their children too.

Key words: in utero, placenta, micro RNAs, cardiovascular disease, diabetes, liver

*Correspondence to:

Sathish Kumar Natarajan, PhD, Assistant Professor, Department of Nutrition and Health Sciences, University of Nebraska-Lincoln;

Lincoln, NE

snatarajan2@unl.edu

Cite This Article: Muthuraj, P.G., Natarajan, S.K. 2020. Fetal Programming in Maternal Obesity. Diabesity 6(3): 36-39. D0I: 10.15562/diabesity.2020.71

\section{Introduction to fetal programming in maternal obesity}

Insufficient nutrition of the mother causes improper growth of the fetus in utero and leads to metabolic syndromes in later stages of life referred to as "the thrifty phenotype hypothesis". 'Similarly, over-nutrition or obesity in the mother can impact the development of the fetus in the womb, leading to increased risks of developing complications as the age of offspring advances. Fetal programming occurs in an unfavorable in utero environment especially affecting critical stages of development and can have a permanent effect that becomes more noticeable phenotypically during adulthood especially in terms of metabolic health. ${ }^{2}$ Childrens of obese mothers are more likely to become obese and this leads to further adverse effects on the offspring's metabolic health during adulthood. ${ }^{3-5}$ Obesity is also known to have multigenerational effects wherein studies show that obese grandparents tend to have obese grandchildren. ${ }^{6,7}$

\section{Placental pathology and other complications in obese mothers and its consequences}

The placenta is an important organ that bridges the gap between the mother and the developing fetus by supplying oxygen and nutrients. Besides this, there are several signaling pathways through which the mother and fetus communicate with each other. Maternal obesity cause placental insufficiency as well as an inflammatory placental environment which affect the developing fetus. A recent study using macaques with high-fat diet and obese dams exhibited adverse placental pathology and had frequent stillbirths. ${ }^{8}$ Similarly, maternal obesity in humans showed increased levels of lipids accumulated in the placenta and results in dysregulation of angiogenesis and also express an pro-inflammatory phenotype and oxidative stress. ${ }^{9}$ Further, obese mothers are more prone to develop gestational diabetes, gestational hypertension and, pre-eclampsia, all of this tend to disturb the normal growth and physiology of the fetus. ${ }^{10}$ Further, unfavorable perinatal and postnatal outcomes such as fetal macrosomia, delivery through cesarean section, and babies maintained in intensive care units are more noticeable with pregnant mothers experiencing unwanted weight gain during gestational time period. ${ }^{11}$

\section{Increased Cardiovascular risks in offsprings born to obese mothers}

The obese maternal environment can also cause untoward effects on the development of fetal heart which leads to various negative cardiovascular outcomes or complications in their later stages of life. In a Finnish epidemiological study, male offspring born to heavier mothers during a period of better nutrition that followed a period of insufficient food supply developed cardiovascular problems in later stages of life. ${ }^{12}$ In a C57BL/6 mice study, offsprings of high fat diet-induced obese female mice were fed with chow diet after weaning and were assessed for their cardiomyocyte development (3-12 weeks of age). Up to 8 weeks of age, cardiomyocyte hypertrophy and expression of cardiac fetal genes were observed and indicating 
adverse cardiac pathology. At 12 weeks of age even though there was no evidence of cardiac muscle hypertrophy, however the authors observed systolic and diastolic dysfunction and upregulation of $\beta 1$ adrenergic receptors, which are the characteristic features of early cardiac failure. ${ }^{13}$ This study again re-iterated the fact that in utero growth environment affects the metabolic health of an individual even with optimum intake of nutrients after birth. In a study with fetuses from high-fat diet-fed baboons, enhanced collagen deposition was seen in the heart and there was dysregulation in the cardiac microRNA expression (miR-133a, -197, miR-30a, miR-499, and miR-451) patterns which contributed to cardiac pathology and abnormalities in the fetuses. ${ }^{14}$ A study in Amsterdam childrens, associated higher pre-pregnancy BMI of mothers showed increased blood pressure in their children aged 5-6. ${ }^{15}$ Similarly, children born to mothers who had obese BMI during pregnancy developed cardiovascular disorder during later part of their lives and also had a life span below average. ${ }^{16}$

\section{Obese mothers and the incidence of diabestes to their offsprings}

Obesity is always associated with adverse metabolic health patterns, similarly, the obese maternal environment programs the fetus towards metabolic syndrome during adolescence or adulthood. A Scottish epidemiological study (1950-2011) demonstrated that increased incidence of maternal obesity incidence during the period of study. Further, they also showed a significant association between incidence of type I and type II diabetes in offsprings born to obese mothers. ${ }^{17}$ In a study using rodents, researchers found that pregnant rats subjected to a high-fat diet, had reduced expression of adiponectin receptors in the litters and, this affected the overall insulin sensitivity and, ultimately disrupted the metabolic health of offsprings. ${ }^{18}$ Likewise, offsprings born to pregnant mice with an obesogenic diet had disrupted endoplasmic reticulum homeostasis as well as apoptotic and autophagy dysregulation in the pancreas. This study established the molecular mechanism behind insulin resistance and early pancreatic beta-cell dysfunction in offsprings born to obese mothers. ${ }^{19}$

\section{Maternal obesity and offspring gut and liver health}

An energy-dense high fat diet in obese mothers causes excessive fat deposition in the liver. Researchers have showed that diet consumed by the mother impacted male offsprings to a greater extent when compared to female offsprings with increased body weight and higher lipid deposition in the liver. ${ }^{20} \mathrm{~A}$ study performed in mice showed complex interactions among maternal obesity, obesogenic diet after weaning and dysregulation of circadian genes in the liver. These complex interactions result in dysregulated pathways involving liver pro-inflammatory fibrogenic mediators leading to non-alcoholic fatty liver disease and further potential increased risk of complications such as hepatocellular adenocarcinoma at later stages life in the offsprings. ${ }^{21}$ It is well known that the gut microbiome is involved in homeostasis and regulation of nutrients and the perturbance of gut microbial composition is associated with obesity. A study based on the analysis of stool samples of babies at 1 and 6 months of age showed that there was an increased concentration of bacteria which promotes pro-inflammatory in nature rather than protective microflora, in babies from obese moms and again this reinstates the fact that offsprings are influenced by maternal environment even after birth. ${ }^{22}$ Studies with mice show that high fat diet fed dams or dams with transplanted gut microbiota from high fat diet fed mice tend to influence the gut microbial composition of the male pups more significantly than female littermates. ${ }^{20,23}$ Further, western diet results in epigenetic modifications in the children, and a reduction in the methylation levels in the blood samples were observed in babies born to obese women and some of these methylation patterns were even reproducible at adolescence stage. ${ }^{24}$ In summary, maternal obesity affects fetal gut microbiome and liver health through epigenetic mechanisms and increases the risk of metabolic disease in offspring in their latter part of life.

\section{Maternal adiposity affects the health of the progeny in a multifaceted manner from behavioral aspects to immune responses}

There is also compelling evidence that cognitive and psychological disorders are highly prevalent among children from obese women. ${ }^{25}$ An Australian study performed with mothers and children using the Child Behavior Checklist during the age of 5, 8, 10, 14 , and 17, and found higher levels of emotional and behavioral issues among children born to pre-pregnancy obese women. ${ }^{26}$ Another interesting cohort study in the state of Pennsylvania linked maternal obesity to the risk of developing childhood cancer like acute lymphoblastic leukemia and neuroblastoma. ${ }^{27}$ Further, maternal obesity has a profound effect on the immune system of the offsprings. Offsprings born to obese dams maintain an overall low-grade inflammatory state, they are also more susceptible to allergic conditions such as asthma and succumb more easily to infections. ${ }^{28}$ Recently, it has been shown that interferon responses to viral infections could be attenuated by upregulating suppressor of cytokine 
signalling-3 (SOCS-3) in obese individuals due to the high levels of circulating leptin. This could enhance susceptibility to vertical disease transmissions which is in turn correlated to mood-related disorders in offsprings. ${ }^{29-31}$ The reproductive status of the offsring can be profoundly influenced by the obese maternal environment. In an animal study, male offsprings from obese mothers had reduced fertility with features of abnormal sperm motility and oxidative stress. ${ }^{32}$ Similarly in another study, they found that pregnant rats fed with a high-fat diet showed poor maternal care and their female offsprings attained puberty earlier and encountered irregularities in the estrus cycle. ${ }^{33}$ Besides this, the offsprings from high fat diet fed dams tend to be light weight on birth but become obese as the days pass supporting the thrifty hypothesis or Barker hypothesis $^{1}$ and these female offsprings typically had elevated leptin levels. ${ }^{33}$ These studies emphasize the fact that the offspring's prolificacy is negatively influenced by the dam's obese environment.

In conclusion, the maternal in-utero environment not only exerts influence on fetal development within the womb but also during later stages of life: childhood, adolescence and, adulthood. The maternal environment influences the fetus via the placenta, along with the pre-existing risks of obesity and the inflammatory environment within the mother itself. These altered physiological states

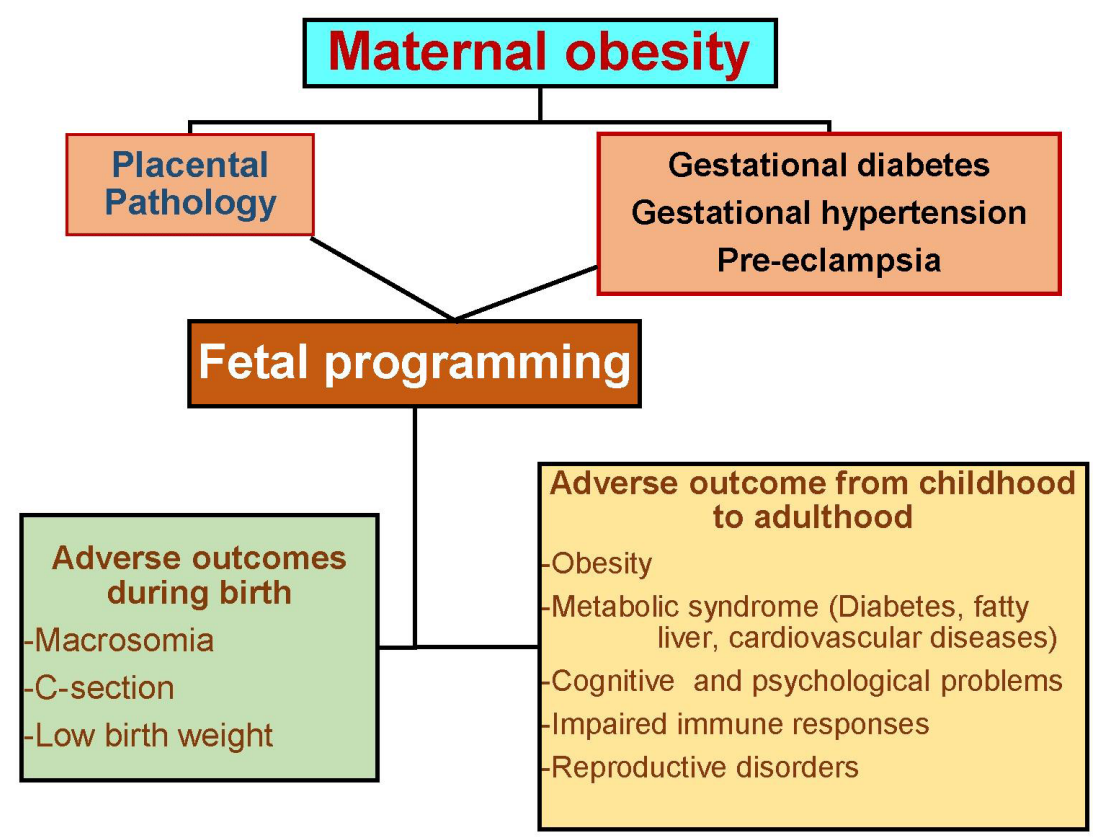

Figure 1 Factors and outcomes associated with maternal obesity in context to fetal programming. Maternal obesity can contribute to factors such as placental pathology, gestational diabetes, gestational hypertension and pre-eclampsia which can trigger fetal programming. This can cause an array of adverse effects on prenatal, postnatal and in adulthood life in the mother bring changes in the epigenetics of the offspring, affecting transcriptional activity of genes responsible for an array of features in the offspring from organs like liver, pancreas to neural tissues with an encompassing overall impact on the metabolic health. Considering obesity as an intervenable risk, lifestyle changes through diet and exercise, as well as bariatric surgery in morbidly obese women who are considering to get pregnant or at their reproductive age, reduces the chances of adverse outcomes in the offspring due to fetal programming.

\section{ACKNOWLEDGEMENT}

The project described was supported by the Nebraska Center for Prevention of Obesity Diseases, the National Institute of General Medical Sciences Grant (P20GM104320 to SKN), the Nebraska Agricultural Experimental Station with funding from the Hatch Act (Accession Number 1014526 to SKN) through the United States Department of Agriculture, National Institute of Food and Agriculture (USDANIFA), the Nebraska Tobacco Settlement Biomedical Research Development Funds and the University of Nebraska-Lincoln. The contents of this manuscript are solely the responsibility of the authors and does not necessarily represent the official views of the National Institutes of Health.

\section{REFERENCES}

1. Hales, C.N. and D.J. Barker, The thrifty phenotype hypothesis. Br Med Bull, 2001. 60: p. 5-20.

2. Marciniak, A., et al., Fetal programming of the metabolic syndrome. Taiwanese Journal of Obstetrics and Gynecology, 2017. 56(2): p. 133-138.

3. Reynolds, R.M., et al., Maternal BMI, parity, and pregnancy weight gain: influences on offspring adiposity in young adulthood. J Clin Endocrinol Metab, 2010. 95(12): p. 5365-9.

4. $\quad$ Page, K.A., et al., Children Exposed to Maternal Obesity or Gestational Diabetes During Early Fetal Development Have Hypothalamic Alterations That Predict Future Weight Gain. Diabetes Care, 2019: p. dc182581.

5. Josey, M.J., et al., Overall gestational weight gain mediates the relationship between maternal and child obesity. BMC Public Health, 2019. 19(1): p. 1062.

6. Davis, M.M., et al., Grandparental and parental obesity influences on childhood overweight: implications for primary care practice. J Am Board Fam Med, 2008. 21(6): p. 549-54.

7. Polley, D.C., et al., Intrafamilial correlates of overweight and obesity in African-American and Native-American grandparents, parents, and children in rural Oklahoma. J Am Diet Assoc, 2005. 105(2): p. 262-5.

8. Frias, A.E., et al., Maternal high-fat diet disturbs uteroplacental hemodynamics and increases the frequency of stillbirth in a nonhuman primate model of excess nutrition. Endocrinology, 2011. 152(6): p. 2456-64.

9. Saben, J., et al., Maternal obesity is associated with a lipotoxic placental environment. Placenta, 2014. 35(3): p. 171-177.

10. Leddy, M.A., M.L. Power, and J. Schulkin, The impact of maternal obesity on maternal and fetal health. Rev Obstet Gynecol, 2008. 1(4): p. 170-8. 
11. Ovesen, P., S. Rasmussen, and U. Kesmodel, Effect of prepregnancy maternal overweight and obesity on pregnancy outcome. Obstet Gynecol, 2011. 118(2 Pt 1): p. 305-12.

12. Forsen, T., et al., Mother's weight in pregnancy and coronary heart disease in a cohort of Finnish men: follow up study. BMJ, 1997. 315(7112): p. 837-40.

13. Blackmore, H.L., et al., Maternal diet-induced obesity programs cardiovascular dysfunction in adult male mouse offspring independent of current body weight. Endocrinology, 2014. 155(10): p. 3970-80.

14. Maloyan, A., et al., Identification and comparative analyses of myocardial miRNAs involved in the fetal response to maternal obesity. Physiol Genomics, 2013. 45(19): p. 889-900.

15. Gademan, M.G.J., et al., Maternal Prepregnancy Body Mass Index and Their Children's Blood Pressure and Resting Cardiac Autonomic Balance at Age 5 to 6 Years. Hypertension, 2013. 62(3): p. 641-647.

16. Reynolds, R.M., et al., Maternal obesity during pregnancy and premature mortality from cardiovascular event in adult offspring: follow-up of 1323275 person years. Bmj-British Medical Journal, 2013. 347.

17. Lahti-Pulkkinen, M., et al., Consequences of being overweight or obese during pregnancy on diabetes in the offspring: a record linkage study in Aberdeen, Scotland. Diabetologia, 2019. 62(8): p. 1412-1419.

18. Hou, M., et al., A high-fat maternal diet decreases adiponectin receptor-1 expression in offspring. J Matern Fetal Neonatal Med, 2015. 28(2): p. 216-21.

19. Soeda, J., et al., Maternal obesity alters endoplasmic reticulum homeostasis in offspring pancreas. J Physiol Biochem, 2016. 72(2): p. 281-91.

20. Wankhade, U.D., et al., Maternal High-Fat Diet Programs Offspring Liver Steatosis in a Sexually Dimorphic Manner in Association with Changes in Gut Microbial Ecology in Mice. Scientific Reports, 2018. 8(1): p. 16502.

21. Mouralidarane, A., et al., Maternal obesity programs offspring non-alcoholic fatty liver disease through disruption of 24-h rhythms in mice. International Journal of Obesity, 2015. 39(9): p. 1339-1348.

22. Collado, M.C., et al., Effect of mother's weight on infant's microbiota acquisition, composition, and activity during early infancy: a prospective follow-up study initiated in early pregnancy. Am J Clin Nutr, 2010. 92(5): p. 1023-30.

23. Bruce-Keller, A.J., et al., Maternal obese-type gut microbiota differentially impact cognition, anxiety and compulsive behavior in male and female offspring in mice. PloS one, 2017. 12(4): p. e0175577-e0175577.
24. Sharp, G.C., et al., Maternal BMI at the start of pregnancy and offspring epigenome-wide DNA methylation: findings from the pregnancy and childhood epigenetics (PACE) consortium. Human Molecular Genetics, 2017. 26(20): p. 4067-4085.

25. Van Lieshout, R.J., Role of maternal adiposity prior to and during pregnancy in cognitive and psychiatric problems in offspring. Nutr Rev, 2013. 71 Suppl 1: p. S95-101.

26. Van Lieshout, R.J., M. Robinson, and M.H. Boyle, Maternal pre-pregnancy body mass index and internalizing and externalizing problems in offspring. Can J Psychiatry, 2013. 58(3): p. 151-9.

27. Stacy, S.L., et al., Maternal Obesity, Birth Size, and Risk of Childhood Cancer Development. American Journal of Epidemiology, 2019. 188(8): p. 1503-1511.

28. Wilson, R.M. and I. Messaoudi, The impact of maternal obesity during pregnancy on offspring immunity. Mol Cell Endocrinol, 2015. 418 Pt 2: p. 134-42.

29. Simanek, A.M. and H.C.S. Meier, Association Between Prenatal Exposure to Maternal Infection and Offspring Mood Disorders: A Review of the Literature. Current Problems in Pediatric and Adolescent Health Care, 2015. 45(11): p. 325-364.

30. Almond, M.H., et al., Obesity and susceptibility to severe outcomes following respiratory viral infection. Thorax, 2013. 68(7): p. 684-686.

31. Godfrey, K.M., et al., Influence of maternal obesity on the long-term health of offspring. The lancet. Diabetes \& endocrinology, 2017. 5(1): p. 53-64.

32. Santos, M., et al., Adult exercise effects on oxidative stress and reproductive programming in male offspring of obese rats. Am J Physiol Regul Integr Comp Physiol, 2015. 308(3): p. R219-25.

33. Connor, K.L., et al., Nature, nurture or nutrition? Impact of maternal nutrition on maternal care, offspring development and reproductive function. J Physiol, 2012. 590(9): p. $2167-80$

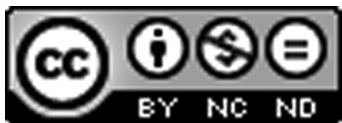

This work is licensed under a Creative Commons Attribution-Non Commercial-No Derivatives 4.0 International License. To view a copy of this license, visit http://creativecommons.org/licenses/by-nc-nd/4.0/ 